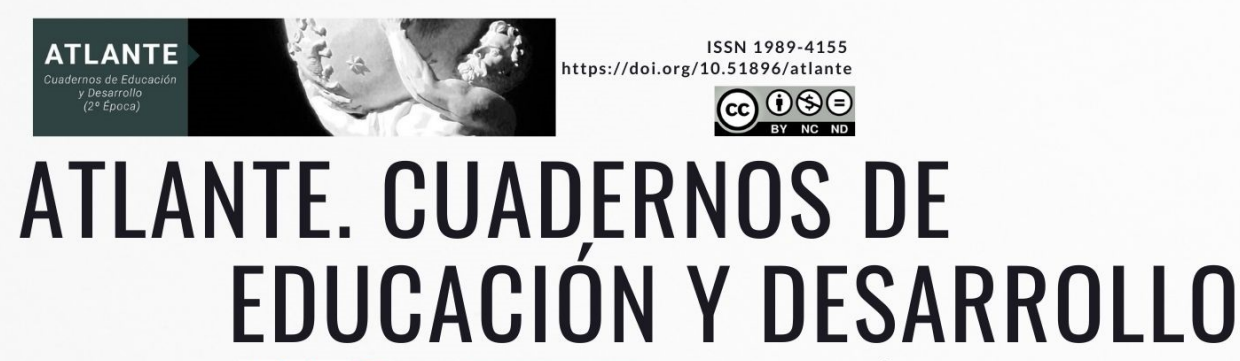

latindex @ideAs EconPapers ODialnet mIAR IInDICEs

\title{
LA CONTRIBUCIÓN DE LAS TIC A LA MEJORA DEL PROCESO DE ENSEÑANZA- APRENDIZAJE DE LENGUAS EXTRANJERAS: APROXIMACIONES TEÓRICAS Y ESTRATEGIAS DIDÁCTICAS
}

\author{
Abidat Fella \\ Universidad Abou El Kacem Saadaalah-Alger2 \\ Doctoranda y profesora temporal en Didáctica en la Universidad de Argel II \\ Email: fella ab@outlook.com \\ Dr. Berraghda Loucif Rabéa \\ Universidad Abou El Kacem Saadaalah- Alger2 \\ Email:r.berraghda@hotmail.fr
}

Para citar este artículo puede utilizar el siguiente formato:

Abidat Fella y Berraghda Loucif Rabéa: "La contribución de las TIC a la mejora del proceso de enseñanza-aprendizaje de lenguas extranjeras: aproximaciones teóricas y estrategias didácticas", Revista Atlante: Cuadernos de Educación y Desarrollo, ISSN: 1989-4155 (vol 13, № 6 julio-septiembre 2021, pp. 122-135). En línea:

https://doi.org/10.51896/atlante/LIED6325

\section{RESUMEN}

En la actualidad, con la hegemonía de las Tecnologías de la Información y de la Comunicación en todos los ámbitos de la vida incluida la educación, se ha vuelto necesaria una readecuación de los procedimientos de aprendizaje. En el área de las lenguas extranjeras, el uso de la tecnología ha permitido que los estudiantes tengan acceso a un conjunto de nuevas herramientas y recursos que contribuyen a la mejora de sus habilidades en la lengua objeto de estudio. El objetivo del presente artículo es establecer en qué medida las tecnologías de la información y de la comunicación impactan en el proceso de enseñanza-aprendizaje de lenguas extranjeras. A partir de una perspectiva conceptual, se brinda unos aportes teóricos relacionados con la temática, así como unas estrategias didácticas que posibilitan la mejora del aprendizaje de lenguas. De igual forma, se proporciona una panorámica de la importancia y la influencia de estas nuevas tecnologías en la educación en general y en el aprendizaje de lenguas extranjeras en particular, además de esto, se ponen de manifiesto las posibilidades, problemas, luces y sombras de la incorporación de las TIC a la enseñanza de lenguas. Por ello, para la realización del presente artículo se basa en una metodología documental a través de la recopilación de 
datos de libros y estudios relacionados con la temática, por lo que el método utilizado es tipo analíticosintético donde se analiza lo antes expuesto. Entre los resultados se evidencia que las tecnologías influyen positivamente en el aprendizaje de lenguas.

Palabras claves: TIC, enseñanza, aprendizaje, lenguas extranjeras, educación.

\title{
THE CONTRIBUTION OF ICT TO THE IMPROVEMENT OF THE TEACHING-LEARNING PROCESS OF FOREIGN LANGUAGES: THEORETICAL APPROACHES AND DIDACTIC STRATEGIES
}

\begin{abstract}
Nowadays, with the hegemony of information and communication technologies in all areas of life including education, it has become necessary to remold our previous learning procedures. In the area of foreign languages, the use of technology aloud the students to gain access to a set of new tools and resources that contribute to the improvement of their skills in the target language. The objective of this article is to establish to what extent information and communication technologies affect the teachinglearning process of foreign languages. From a conceptual perspective, we pretend provide theoretical contributions related to the subject, as well as some didactic strategies that make it possible to improve language learning. Similarly, we pretend provide an overview of the importance and influence of these new technologies in education in general and in foreign language learning in particular. In addition to this, we will highlight the possibilities, problems, lights and shadows of the incorporation of ICT into the teaching of languages. Therefore, for the realization of this article we are based on a documentary methodology through the compilation of data from books and studies related to the subject, so the method used is analytical-synthetic type where the above is analyzed. Among the results, it is evidenced that technologies positively influence language learning.
\end{abstract}

Key words: ICT, teaching, learning, foreign languages, education.

\section{INTRODUCCIÓN}

Las tecnologías de la información y de la comunicación ${ }^{1}$ están presentes en cada esfera de la sociedad y en todos nuestros procederes actuales, brindando numerosas posibilidades a sus usuarios y facilitando su forma de vivir. Desde los mensajes de texto o de voz, la consulta del correo electrónico, la posibilidad de leer noticias en línea, comprar productos, publicar fotos, descargar música y videos y participar en conferencias a distancia, las nuevas tecnologías han logrado facilitar enormemente nuestras interacciones sociales.

Con estos avances tecnológicos o digitales que han cambiado la manera de difundir y de concebir la sociedad del siglo XXI, la educación y el aprendizaje no pueden mantenerse al margen. Hoy en día estas tecnologías están implantadas en el sector educativo para transformar la enseñanza y promover el

\footnotetext{
${ }^{1}$ Las TIC, en lo adelante.
} 
aprendizaje de los niños y jóvenes, en quienes estas nuevas tecnologías han impactado de forma positiva al usarlas adecuadamente. F. Blázquez (2001) apunta acerca de esto:

La clave de la sociedad actual es, de uno u otro modo, la capacidad de procesar la ingente cantidad de información de que disponemos, gracias al desarrollo de las nuevas tecnologías, transformándola en el conocimiento necesario para cambiar nuestro entorno, en la búsqueda de una mayor libertad, igualdad y solidaridad entre los seres humanos, en un mundo que está rompiendo los moldes acuñados en el siglo XX, al haberse mostrado incapaces de resolver el hambre, la guerra, la ignorancia, el racismo, la xenofobia y otros tantos defectos que, en lugar de disminuir con el progreso del ser humano, se acrecientan y hacen más profunda la distancia, a pesar de acercarnos a un mundo sin fronteras. Si seguimos de cerca los cambios que se están produciendo, día tras día, en las formas de comunicarnos entre los seres humanos, debido a los continuos avances tecnológicos en los sistemas de tratamiento y transmisión del conocimiento, llegamos a la conclusión de que los procesos de enseñanza/aprendizaje deben estar en permanente revisión para incorporar los nuevos métodos en el sistema educativo. Dicho trabajo necesariamente debe incorporar todos los recursos humanos implicados en nuestra Región, tarea compleja, pero no imposible, por la excelente predisposición de docentes y dicentes, ávidos de implicarse de lleno en los avances del siglo XXI. (p. 7)

Los jóvenes de hoy, al momento de consultar cualquier información, acuden directamente al Internet, son muy pocos los que prefieren preguntar a sus profesores. Asimismo, hay algunos que piensan que se puede aprender mejor fuera de la escuela pues asistir todos los días a clases magistrales les resulta aburrido. Sin embargo, la implantación de las TIC en la educación no implica solamente cambiar los materiales tradicionales de enseñanza por otros más contemporáneos como el ordenador, Internet, el móvil o pizarras interactivas. Se necesita una renovación de las herramientas que se base en un cambio de la forma de inculcar estos conocimientos por parte de los profesores y adopte una postura y visión diferentes, siempre en constante actualización y evolución que permita a los educadores desarrollar su labor apropiadamente. (Benavides et al., 2011, p.3).

El objetivo que perseguimos a través del presente estudio es determinar de qué manera las Tecnologías de la Información y de la Comunicación impactan en el proceso de enseñanza-aprendizaje de lenguas extranjeras. En las próximas líneas intentamos desarrollar unas reflexiones en torno al concepto TIC, considerado el núcleo del presente artículo, y cómo puede brindar a los estudiantes una nueva forma de aprendizaje. Por supuesto, estos planteamientos levantan nuevos cuestionamientos: ¿cómo las nuevas 
tecnologías ayudan a promover un aprendizaje significativo?, ¿cómo ayudan a los estudiantes a desarrollar sus habilidades lingüísticas?, ¿de qué manera la integración de la tecnología de información y comunicación apunta a la mejora del aprendizaje y motiva a los estudiantes, al convertirlos en los principales actores en el aula?

En este artículo, presentamos unas aproximaciones teóricas acerca de las TIC, proporcionando al alumnado unas estrategias y aplicaciones para la mejora del proceso de adquisición de idiomas. Por lo que, para su realización nos basamos en una metodología documental a través de la recopilación de datos y informaciones de libros y estudios que corresponden con el tema principal

\section{Tecnología y educación}

La educación hoy en día goza de muchas oportunidades y posibilidades para que continúe su avance y evolución, de la forma sin precedentes en que lo hace. El ilimitado potencial de la tecnología como agente de cambio nunca ha sido más evidente.

Actualmente, muy pocos estudiantes llegan a la universidad sin poseer tecnologías móviles potentes, ya sean computadoras portátiles, tabletas o teléfonos inteligentes. Estas tecnologías tienen enormes capacidades informáticas y ofrecen acceso a miles de aplicaciones, a menudo gratuitas, fácilmente descargables a través de Internet. Los alumnos acceden con regularidad a las redes y a plataformas como Facebook, iTunes, YouTube, Skype, Blog y Twitter. La tecnología educativa requiere, entonces, integrar las tan diversas herramientas tecnológicas en el proceso de aprendizaje para facilitar y promover la educación. Esto constituye la integración de las TIC en el proceso educativo. (Fox, 2011, p1).

El concepto de Tecnología Educativa apareció en el año 1941 en la Encyclopedia of Educational Research, como una disciplina pedagógica en Norteamérica, no obstante, sus raíces se dan en la didáctica que por su parte hasta los años sesenta fue articulada como campo de estudio. (Muñoz, 2002, p.69).

La tecnología educativa ha pasado por diversas etapas, en las que se han generado muchos cambios que han metamorfoseado tanto los contenidos inculcados como la metodología utilizada.

La irrupción de las Tecnologías de la Información y de la Comunicación en los siglos XX y XXI, ha provocado una revolución en todo sentido, especialmente en los campos de enseñanza/ aprendizaje. J. Cabero (2003) define la Tecnología Educativa:

La tecnología educativa se nos ha presentado a lo largo de su historia como una disciplina integradora, viva, polisémica, contradictoria y significativa, aludiendo con ello a la importancia que han tenido las transformaciones en las que se ha visto 
inmersa y las diversas formas de entenderla con la que nos encontramos. (p. 23)

J. Cabero (2007ª $)$ explica que la tecnológica educativa puede ser integradora, dado que aúna diversas corrientes científicas y disciplinas como es el caso de la física e ingeniería o la psicología y la pedagogía, sin perder de vista a la teoría de la comunicación. Puede también ser viva, al encontrarse en el continuo y constante cambio y evolución que se produce en las ciencias que la sustentan. Es polisémica en el sentido de que a lo largo de su historia ha recibido diversas definiciones y significados que cambian de acuerdo al contexto de su utilización, puede ser cultural, social y científica. Su carácter contradictorio se debería a la provocación de oposiciones y confrontaciones radicales. Y es, por supuesto, significativa debido a la importancia que ha alcanzado y que puede constatarse en el volumen de congresos, revistas, publicaciones y asociaciones.

\section{Concepto TIC}

Las siglas TIC hacen referencia a las Tecnologías de la Información y de la Comunicación (en inglés Information and Communication Technologies o ICT). Por su parte, el acrónimo T.I.C.E. (Tecnologías de la Información y la Comunicación en la Educación o en la Enseñanza) suele ser usado pero no es totalmente aceptado porque se estima que no existe una tecnología diseñada solamente para este uso sino que se adaptan a cada ámbito. (Torres, 2001, p.85).

Por ello, las TIC son herramientas tecnológicas que abarcan desde los más tradicionales artefactos como la radio, el cine, la televisión, el video y las redes telefónicas, hasta la invención de las tecnologías modernas como el internet y la web 2.0 y todos los servicios que ofrecen. Los autores han definido de manera diferente este término.

Por lo que, de acuerdo con Cabero Almenara citado por Ayala y S. González (2015):

Las TIC giran en torno a tres medios básicos: la microelectrónica, la informática y las telecomunicaciones; pero giran, no solo de forma aislada sino, lo que es más significativo, de manera interactiva, lo que permite conseguir nuevas realidades comunicativas. (p. 28)

En relación a lo expuesto por Cabero (2015), estas nuevas tecnologías se caracterizan por tener un carácter interactivo e interconectado lo que permite conseguir nuevas realidades comunicativas y un intercambio de información de manera eficiente.

Javier Echeverría (2001), por su parte, afirma de las TIC que:

Cuando digo 'tecnologías', me estoy refiriendo, simple y llanamente, al teléfono, a la televisión, a las tarjetas de crédito, a las redes telemáticas 
como Internet, a las tecnologías multimedia como los cederrón, los DVD, es decir, los discos de archivo digitales y electrónicos, o las enciclopedias multimedia, a los videojuegos y a la realidad virtual, tecnología esta última que también debe ser tenida en cuenta y de la que forman parte los nuevos cascos estereoscópicos o las gafas igualmente estereoscópicas del cine Imax, unos artefactos superpuestos al cuerpo humano capaces de generar percepciones y sensaciones reales. (p. 1).

Desde esta perspectiva, se puede decir que la tecnología abarca un conjunto de artefactos que conforman un nuevo espacio social, un espacio electrónico que está suscitando grandes cambios en todas las esferas de la vida, permite a los usuarios acceder a sus aplicaciones y datos en tiempo real, en cualquier dispositivo, independientemente del lugar donde están.

Daniel Cabrera (2006) señala que estas tecnologías son:

Un conjunto heterogéneo de aparatos, discursos e instituciones que se instituyen como realidades posibles y deseables en el marco de matrices imaginarias que tanto pueden legitimar el orden social actual como proporcionar una dimensión utópica que canalice las necesidades de transformación. (p. 154).

A partir de la definición de Cabrera (2006) que identifica a las nuevas tecnologías como un conjunto de aparatos, dispositivos, instituciones y discursos variados y diferentes que materializan el imaginario de la sociedad actual.

\section{Ventajas e inconvenientes de las TIC}

\subsection{Las ventajas de las TIC}

Gracias a las TIC el mundo parece haberse vuelto más pequeño y aparenta estar cada vez más interconectado. Sin embargo, no se debe pasar por alto que las tecnologías son un arma de doble filo y que, a lo largo de la historia, han presentado tantas ventajas como han sido generadoras de inconvenientes. Entre los beneficios que las TIC brindan, tanto en el área de la educación como en los otros diferentes ámbitos de la vida, Área Moreira destaca (2009, p. 7):

- Las TIC facilitan la comunicación entre las personas, rompiendo las barreras geográficas y temporales mediante diferentes formas, ya sea orales por el teléfono por ejemplo o por escrito a través el correo electrónico, redes sociales.

- La comunicación con las TIC puede ser sincrónica o asincrónica. 
- Las TIC permiten un fácil acceso a gran cantidad de información propiciada por el auge de los medios de comunicación; estos medios permiten a las personas estar siempre al día, informados de todo lo que sucede a su alrededor.

- $\quad$ El acceso instantáneo a bibliotecas o instituciones.

- $\quad$ Con las tecnologías se vuelve más fácil realizar consultas o transferencias comerciales en una institución bancaria, por ejemplo.

- $\quad$ Estas tecnologías afectan también al mundo laboral y aumentan las posibilidades de trabajo, con la aparición del teletrabajo o trabajo a distancia mediante ordenador, por ejemplo.

En cuanto a su repercusión en la educación, Cabero (2007, p. 13) destaca los siguientes beneficios:

- Amplía la oferta educativa.

- Elimina las barreras espacio-temporales.

- Flexibiliza el aprendizaje.

- Incrementa las modalidades comunicativas.

- Favorece tanto el aprendizaje independiente como el cooperativo.

- Favorece los espacios y entornos interactivos.

- Mejora las posibilidades de orientación y tutorización de los estudiantes.

\subsection{Los inconvenientes de las TIC}

Entre las desventajas o los inconvenientes que estas tecnologías de la información y de la comunicación generan, Moreira (2009, p. 8) cita:

- Alteración del funcionamiento de los ordenadores u otro tipo de dispositivos, provocada por los virus informáticos.

- La supremacía o el predominio de la cultura occidental sobre el resto del mundo debido a la globalización y la masificación de los medios de comunicación en todo lo que atañe a ropa, música, comida, juegos, películas, etcétera.

- La violación de la privacidad y la seguridad, al incluir datos personales de los usuarios.

\section{Los problemas educativos vinculados con las TIC}

Según Moreira (2009, p. 9), los problemas educativos asociados al uso de las TIC son los siguientes:

- Inadaptación a todas las innovaciones tecnológicas acaecidas en muy corto tiempo con una excesiva velocidad.

- Analfabetismo tecnológico, la alfabetización tecnológica hoy en día es de importancia primordial para saber manejar estos dispositivos. 
- Saturación de la información, con esta inconmensurable cantidad de información encontrada en la red se vuelve necesario saber distinguir lo importante de lo accesorio.

- Dificultades para entender las formas hipertextuales, existen diferentes maneras de acceso a la misma información.

- Desfase o desajuste de los sistemas educativos, por la lenta inclusión y adaptación de los avances de la tecnología a sus sistemas.

\section{Las TIC y sus modalidades de aprendizaje}

Las metodologías de enseñanza y aprendizaje de lenguas extranjeras están en constante cambio debido al auge de las nuevas tecnologías y a su integración en el currículo. Esto ha propiciado la aparición de diversas modalidades educativas o estrategias didácticas que generan cambios en el paradigma educativo tradicional.

Por modalidades educativas se hace referencia a los medios, procedimientos, estrategias didácticas que se llevan a cabo durante el proceso de enseñanza aprendizaje o simplemente para realizar la acción educativa. Así que, una nueva modalidad implica cambios en el paradigma educativo el antes establecido. Uno de éstos lo generó el auge de las TIC que requieren una reestructuración de la manera de concebir el proceso de enseñanza-aprendizaje. De las diversas clasificaciones de las modalidades educativas, la más aceptada la divide en tres grupos: Presencial, No presencial (abierta y a distancia) y Mixta. (Ramos, 2006, p.6).

Entre las diferentes modalidades de enseñanza-aprendizaje que las TIC han permitido, Consuelo Belloch (2012, p.1) cita:

$\bullet$

La enseñanza presencial: con el fin de mejorar la calidad del aprendizaje en la presente era, se insertan y se usan estas nuevas tecnologías en el aula tradicional como herramientas facilitadoras del proceso educativo.

$\bullet$

La enseñanza no presencial (a distancia o virtual): llamada E- learning, es un tipo de educación que no requiere la asistencia presencial a clase, todo el proceso educativo se lleva exclusivamente por Internet.

La enseñanza semipresencial o mixta: llamada también blended learning o B-learning, es la combinación de las dos modalidades anteriores, o sea, el aprendizaje cara a cara con la modalidad a distancia.

La evolución de las TIC y su integración en el proceso educativo ha permitido la introducción de diferentes modalidades en la enseñanza, entre las que destacan las modalidades presenciales, virtuales, mixtas, ubicuas, personalizadas o móviles.

\section{Beneficios de la enseñanza de lenguas extranjeras, asistida por las TIC}


El dominio de lenguas extranjeras en el milenio actual, una era cada vez más globalizada en la que todo está interconectado, se ha trasformado en el pasaporte que autoriza la interacción con otras culturas. E extraordinario desarrollo que las tecnologías de la información y la comunicación ha experimentado abarca los ámbitos de la educación y la enseñanza de lenguas. (Carmona, 2013, p. 165).

La enseñanza-aprendizaje de lenguas no ha sido inmune a los cambios y los avances producidos por las nuevas tecnologías. El nuevo desarrollo tecnológico se ha ganado un lugar dentro del ámbito de la enseñanza, al ser no solamente fuente de recursos sino también un medio de aprendizaje sin igual.

Las TIC permiten a los estudiantes y usuarios de una lengua usarla y practicarla en contextos reales y auténticos a través de Internet, lo que resulta un elemento altamente motivador para el aprendiz. Entre los materiales y herramientas que ofrecen estas posibilidades podemos encontrar periódicos en línea, webcasts, podcasts o, videos de YouTube.

Antes de la emergencia de las TICs, se solía trabajar con materiales tradicionales como la pizarra, el libro, la tiza y mapas fotos para ilustrar lo enseñado. Actualmente, el acceso instantáneo a estos materiales mediante las redes hace que todo el proceso de enseñanza-aprendizaje resulte mucho más fácil. A esto se añade la disponibilidad de diferentes herramientas y aplicaciones para practicar la pronunciación y la entonación, mediante conversaciones con otros a través de plataformas como Skype que permiten hablar, escribir y verse simultáneamente en línea, economizando tiempo y energía.

Las TIC permiten a los usuarios de la lengua escribir, leer, hablar, escuchar, reaccionar e intervenir en una conversación como parte del proceso educativo. Esto los motiva a seguir hablando y deshacerse de todos los posibles problemas o complejos que forman parte del proceso de la comunicación. Asimismo, el rol del profesor ha evolucionado gracias a las modalidades semipresencial, combinada o mixta que existen hasta permitirle convertirse en un mediador. El estudiante, por su parte, alcanza un rol activo como protagonista en su aprendizaje. Sangrá y González (2004) citado por Cabero Almenara (2005) explican sobre este punto que:

El estudiante también deberá aprender a modificar su actitud y el rol que ha desarrollado hasta ahora. Tendrá que adoptar un papel activo, ya que tendrá que convertirse en el protagonista real de su proceso de aprendizaje, mientras que el educador, el profesor, como ya hemos dicho, cambia su función y se convierte en el dinamizador, el guía, el encargado de facilitar el proceso de aprendizaje del estudiante. Pero para ello, el profesorado deberá hacer el esfuerzo de entender al estudiante, de acompañarlo en la entrada a un nuevo contexto formativo, más abierto, menos normativo, más libre y, por lo tanto, menos protector. (p. 89). 
El uso de las TIC en el blended learning ofrece muchas posibilidades y facilita la tarea a los profesores de lengua gracias a las herramientas educativas que permiten seguir la evolución de sus estudiantes y ofrecerles una orientación personal. Con la mejora de los estilos y metodologías de aprendizaje tradicionales, cada estudiante puede llevar su propio ritmo de asimilación en dependencia de directa de sus necesidades y habilidades. (Kumar y Tammelin, 2008, p. 6).

Las herramientas TIC aplicadas en el aula de lenguas que más se conocen son el ordenador, Internet, la radio, el móvil, el tablet $\mathrm{PC}$, el proyector de video y las pizarras digitales, usadas en los centros de lenguas privados.

\section{Herramientas y estrategias de las TIC en la enseñanza - aprendizaje de lenguas extranjeras}

Para Hugo Castellano (2011) las herramientas informáticas, o aplicaciones, abarcan al software como elemento diseñado para la realización de un conjunto de tareas. En las herramientas software se puede hacer una diferenciación entre aplicaciones de escritorio (desktop o stand-alone), las ejecutadas o instaladas en el ordenador y que no requieren conexión para funcionar, y las aplicaciones web cuya ejecución requiere de una conexión a la red.

Entre las herramientas TIC utilizadas durante el proceso de enseñanza-aprendizaje en general, encontramos aplicaciones para la búsqueda, comprensión, aprendizaje y difusión, así como para la comunicación e interacción social. Estas últimas pueden ser usadas dentro o fuera del aula de lenguas, como parte de las modalidades presenciales o semipresenciales. Moreira (2009) y Ayala y González (2015) recogen algunas de las principales herramientas utilizadas:

- Sitios Web de publicación compartida (YouTube, Wordpress, Flickr, Slideshare, Blogspot): son recursos que sirven para buscar, publicar, y compartir informaciones o ficheros.

- Bibliotecas o revistas virtuales: son plataformas o sitios web que ponen al servicio del usuario información o revistas y libros de diferentes temáticas.

- Aula Virtual: es un recurso que ofrece a los estudiantes una enseñanza en línea o a distancia y, por ende, facilita la comunicación y la interacción entre profesor y estudiante.

- Plataformas de software para aulas virtuales (Moodle, Ccaroline, Chamilo, Blackboard): son portales utilizados para ofrecer clases, actividades y cualquier tipo de información a los estudiantes a distancia o de forma semi-presencial, o sea continuar la clase presencial en línea.

- Videoconferencia: es un sistema que permite establecer una comunicación con una o varias personas que se encuentran geográficamente alejadas, de manera bidireccional y sincrónica. Permite la interacción entre usuarios y la realización de exposiciones o charlas con expertos sobre cualquier tema, etcétera.

- Correo electrónico: es un recurso para mandar y recibir mensajes de manera electrónica por medio de Internet. 
- Chats: es un servicio que facilita la comunicación entre personas, tiene un carácter asincrónico por lo que la conversación se mantiene en tiempo real. Para llevarlo a cabo los usuarios deben conectarse a la misma hora.

- Foro: Es una herramienta usada en la red, o sea, un espacio virtual en el que los estudiantes o los usuarios pueden mantener conversaciones, enviar mensajes o participar en debates.

- Webquest: es una aplicación basada en la estrategia de aprendizaje por descubrimiento, una maniobra didáctica o actividad de investigación de informaciones procedentes del internet, guiada por el profesor.

- Wiki: es un sitio web abierto al público y que permite la edición y el cambio de su contenido por parte de sus usuarios, bajo control del administrador.

- Blogs, Weblogs o bitácoras: son recursos que permiten al usuario crear su diario personal. El blog es un espacio en el que el estudiante puede impartir contenidos interesante sobre el aprendizaje de lenguas, ingresando imágenes o videos. Puede, también, ser creado por el profesor para facilitar el aprendizaje de sus discentes, así cada vez que alguien ingresa a este blog o bitácora encuentra todo lo publicado por el propietario, ya sea profesor o estudiante.

- Software de presentación multimedia (PowerPoint): es un programa utilizado en el aula para hacer presentaciones mediante diapositivas, incluyendo textos, esquemas, gráficos, fotos, animaciones y videos.

- Redes sociales (Facebook, Twitter, entre otras): son herramientas de comunicación entre personas y favorecen el aprendizaje y la interacción entre los estudiantes a través del juego, los test y otras aplicaciones.

Añadimos, a los recursos antes citados, otras herramientas e instrumentos que contribuyen al aprendizaje y a la profundización del conocimiento de una lengua:

- Google Docs: sirve para crear y compartir documentos.

- Issuu: es un servicio utilizado para visualizar y descargar material digitalizado, como libros y artículos.

- Slideshare: para compartir presentaciones y documentos.

- Youtube: una aplicación para ver y compartir videos.

- Skype: herramienta que permite la comunicación mediante imagen y sonido.

- JClic: un entorno que puede ser utilizado para realizar actividades educativas como puzles y crucigramas.

- Prezi: una aplicación para crear, compartir y visualizar presentaciones.

- Hot Potatoes: es un software creado para realizar actividades y ejercicios en línea. 
En conjunto con todas estas herramientas, existe una multitud de aplicaciones descargables para móvil que son de gran utilidad en el aprendizaje de lenguas extranjeras, desde el nivel principiante hasta el más avanzado. Las más conocidas son BUSSU, RossetaStone y Duolingo entre otros.

Todas estas posibilidades existen gracias al desarrollo que han tenido las TIC a lo largo de la historia de la tecnología y los servicios de telecomunicaciones. No obstante, para poder manejar y usar adecuadamente estas tecnologías es necesario que tanto los profesores como los estudiantes cuenten con una alfabetización tecnológica, o sea, una instrucción y conocimientos sobre el uso y el manejo de ellas.

La enorme diferenciación que existe entre los que tienen acceso a internet y los que no, sobre todo con la evolución de estas herramientas y el efecto de la globalización, ha generado problemas y ha creado una brecha enorme entre las personas. Con el invento, en su momento, de la radio y la televisión se pensaba que los libros, la lectura y la escritura serían reemplazados. Lo mismo ocurre con el auge de estas nuevas tecnologías que se cree reemplazarán a las antiguas formas de comunicación y de enseñanza-aprendizaje.

Las TIC son una innovación que goza de cierta prominencia y prestigio, permiten cambios determinantes en todos los ámbitos de la vida humana y, sin duda, brindan herramientas y posibilidades sorprendentes. Sin embargo, el fenómeno de brecha digital creado por la multitud de desajustes existentes en la sociedad, personas alfabetizadas versus analfabetas, ricos versus pobres y problemas generacionales, solo se ve aumentado con estos nuevos avances.

Nos rodeamos constantemente de la tecnología por lo que se requiere desarrollar nuestros conocimientos y habilidades para saber usar las nuevas herramientas adecuadamente y para formar a personas digitalmente alfabetizadas, que suban a esta ola digital y continúen esta nueva corriente.

En lo relacionado con el ámbito educativo es imprescindible que tanto los profesores como los estudiantes tengan acceso a las TIC, dentro y fuera del aula. Es importante, además, que cuenten con una formación digital que reduzca el analfabetismo tecnológico. Sobre este tema, opina Rodríguez Gallardo (2006):

La gente más educada está más abierta a las innovaciones en cualquier campo. Las personas que tienen bajos niveles educativos tienden a ser más tradicionales y, por lo tanto, presentan mayor resistencia al cambio y en nuestro caso a la utilización de los instrumentos tecnológicos que les permitan informarse. (p. 226).

\section{CONCLUSIÓN}


Luis Guillermo Restrepo Rivas (1999) opina que «Las TIC no son simplemente máquinas, ni mentes ni cerebros, sino interactuadores de habilidades, ideas y servicios y de colaboraciones infinitas entre los seres humanos.» (p. 2).

No se puede negar que, a pesar de la importancia de estas herramientas, existe una flagrante ignorancia en cuanto al uso de las nuevas tecnologías y la brecha entre quienes tienen acceso a ellas y quienes no siguen su aumento.

Estas tecnologías de la información y de la comunicación son fuerzas omnipresentes en todas las esferas de la sociedad y la educación, en la que el ámbito de enseñanza-aprendizaje de lenguas extranjeras adquiere un rol de importancia. Actualmente, la enseñanza de lenguas asistida por las nuevas tecnologías se ha vuelto una apremiante necesidad debido a su amplio potencial didáctico y su papel en la mejora del aprendizaje y motivación de los estudiantes, convirtiéndolos en los principales actores de su aprendizaje.

El ámbito de la enseñanza de lenguas hoy en día está en constante cambio y evolución. Los avances deben tomar en cuenta, por un lado, a la innovación y evolución de las metodologías de enseñanza de lenguas y, por otro, al desarrollo que están teniendo estas tecnologías en la educación. Las TIC nos empujan a cambiar la visión arcaica que siempre hemos tenido sobre la didáctica de lenguas y, a pesar de que la tecnología debe ser manejada con cuidado, el uso adecuado de estos apoyos facilitan las tareas pedagógicas del profesorado y del estudiantado.

En referencia a los cuestionamientos planteados se puede decir que las herramientas tecnológicas presentadas en este trabajo ayudan a promover un aprendizaje significativo ya que disponen de informaciones de todo tipo que permiten a los estudiantes desarrollar sus habilidades lingüísticas. Por lo tanto, el uso de las TIC dentro o fuera del aula de lenguas extranjeras contribuye a dinamizar las metodologías de enseñanza-aprendizaje con técnicas diferentes al modelo tradicional. Por otra parte, promueven la autonomía, protagonismo del estudiante. Su integración apunta a la mejora del aprendizaje y motiva a los estudiantes convirtiéndoles en los principales actores en el aula.

Finalmente, después de presentar la importancia que han tenido estos dispositivos se puede decir que el uso de las TIC ha mejorado la calidad de la enseñanza de lenguas extranjeras.

\section{BIBLIOGRAFÍA}

Área Moreira, M. (2009). Introducción a la tecnología educativa. Universidad de la Laguna.

Ayala, E. y Gonzales Sánchez, S. (2015). Tecnologías de la Información y la Comunicación. Fondo Editorial de la UIGV.

Belloch, C. (2012). Unidad de Tecnología Educativa (UTE). Universidad de Valencia. 
Benavides, Á.; Bairon, A.; Córdoba, E.; Rodríguez, E.; Erazo, E.; Greis, S. y Bolaños, S. (2011). Crear y publicar con las TIC en la escuela. Universidad del Cauca: Colombia.

Blázquez Entonado, F. (2001). Sociedad de la información y educación. Consejería de Educación, Ciencia y Tecnología. Junta de Extremadura.

Cabero Almenara, J. (2003). Replanteando la tecnología educativa. Comunicar, (21) ,23-30.

Cabero Almenara, J. (2005). «Las TIC y las universidades: retos, posibilidades y preocupaciones». Revista de la educación superior, 34 (135), pp. 77-100,

Cabero Almenara, J. (2007). «Las nuevas tecnologías en la Sociedad de la Información». In Nuevas tecnologías aplicadas a la educación, número, pp. 1-20, Revista de la Educación Superior Universidad de Sevilla.

Cabero Almenara, J. (2007록. «Tecnología educativa: su evolución histórica y su conceptualización». In Tecnología educativa, número, pp. 13-28, Revista de la Educación Superior Universidad de Sevilla.

Cabrera, D. (2006). Lo tecnológico y lo imaginario. Nuevas tecnologías como creencias y esperanzas colectivas. Editorial Biblos. Buenos Aires.

Carmona, M. P. (2013). «Nuevas tareas para el profesor de español como lengua extranjera: la reflexión sobre su concepción de la enseñanza». Porta Linguarum: revista internacional de didáctica de las lenguas extranjeras, 19, pp. 165-178.

Echeverría, J. (2001). «Sociedad y nuevas tecnologías en el siglo XXI». Conferencia brindada en Málaga, España.

Fox, R. (2011). «Technological practice and change in education». In International Conference on ICT in Teaching and Learning, (es libro, autores), pp. 1-7. Springer, Berlin.

Hugo, M. C. (2011). Integración de la tecnología educativa en aula. Enseñando con las TIC. Cengage Learning. Argentina.

Kumar, S.y Tammelin, M. (2008). «Integrar las TICS en la enseñanza/aprendizaje de segundas lenguas». Una guía para instituciones educativas europeas de Secundaria, Universidad y Educación para adultos.

Muñoz, A. G. V. (2002). Tecnología educativa: Características y evolución de una disciplina. Revista Educación y Pedagogía, (33), 65-87.

Ramos, C. B. (2006). «Acercamiento a las nuevas modalidades educativas en el IPN». Innovación Educativa, 6 (30), pp. 5-16, México.

Restrepo Rivas, L. G. (1999). Las tecnologías de la información y las comunicaciones en la empresa. Medellín.

Rodríguez Gallardo, A. (2006). La brecha digital y sus determinantes. México.

Torres, M. (2001). «Integración de tecnologías multimedia en la enseñanza de lenguas». In Revista Lenguaje, 28, pp. 84-107, Universidad Del Valle. 